\title{
Pseudogout: A Rare Cause of Acute Arthritis Following Arthroscopic Anterior Cruciate Ligament Reconstruction
}

\author{
Mahvash Zaman, $\mathrm{MBChB}^{1}$, Numaera Sabir, $\mathrm{MBChB}^{1}$, Simon Peter Mills, $\mathrm{MBChB}^{1}$, and Charalambos $\mathrm{P}$. \\ Charalambous, FRCS $^{1-3}$ \\ ${ }^{1}$ Department of Orthopaedic, Blackpool Victoria Hospital, Blackpool; ${ }^{2}$ School of Medicine and Dentistry, University of Central Lancashire, Preston; ${ }^{3}$ Institute of \\ Inflammation and Repair, Faculty of Medical and Human Sciences, University of Manchester, Manchester, UK
}

We report a case of an acute pseudogout attack following single-bundle anterior cruciate ligament (ACL) reconstruction in a 35-year-old man. At the initial reconstruction surgery, he was found to have early degenerative changes mainly in the lateral compartment. He presented with acute onset pain and swelling following reconstruction of the ACL. Arthroscopic irrigation was performed and the synovial fluid was positive for calcium pyrophosphate crystals. A pseudogout attack must be considered in the differential diagnosis in cases of acute onset pain and swelling after arthroscopic surgery, especially with the background of degenerative knee changes, and this may signify a poorer long-term outcome.

Keywords: Knee, Arthritis, Pseudogout, Anterior cruciate ligament, Reconstruction

Pseudogout is a condition where crystals of calcium pyrophosphate dihydrate (CPPD) are deposited within a joint ${ }^{1)}$. Calcification of cartilage in the knee is the most common finding as a result of CPPD deposition ${ }^{2}$. Pseudogout classically presents with symptoms of acute or chronic inflammation of a joint ${ }^{3}$, but patients can often be asymptomatic ${ }^{1}$. In a postoperative patient, the presentation of an attack of pseudogout can be identical to that of a septic arthritis ${ }^{1)}$. We present a report of a rare case of pseudogout attack in a patient after anterior cruciate ligament (ACL) reconstruction. The common features, presentation and diagnostic difficulties are discussed.

Received August 7, 2013; Revised (1st) March 24, 2014;

(2nd) November 10, 2014; (3rd) February 21, 2015; Accepted June 1, 2015

Correspondence to: Simon Peter Mills, MBChB

Department of Orthopaedic, Blackpool Victoria Hospital, 38 Whinney

Heys Road, Blackpool, Lancashire FY3 8NR, UK

Tel: +44-01253-655983, Fax: +44-01253-303530

E-mail: bcharalambos@hotmail.com

This is an Open Access article distributed under the terms of the Creative Commons Attribution Non-Commercial License (http://creativecommons.org/licenses/by-nc/4.0/) which permits unrestricted non-commercial use, distribution, and reproduction in any medium, provided the original work is properly cited.

\section{Case Report}

A 35-year-old man underwent ACL reconstruction of the right knee with hamstring tendons and partial lateral meniscectomy for a meniscal tear. Magnetic resonance imaging scan showed moderate osteoarthritic changes and a subchondral cyst in the central portion of the lateral femoral condyle (Fig. 1). At arthroscopy, he was found to have degenerative chondral changes in the lateral compartment, mainly on the tibial side. The medial meniscus showed evidence of previous partial meniscectomy that had been performed six years previously. He responded well initially and underwent a physiotherapy rehabilitation program.

At six weeks after surgery, he presented with a two-day history of pain and swelling in the knee. He did not report any locking or giving way, or a new injury. Clinically, he had a severe effusion in the knee, but he was able to bear weight with some discomfort. He had a limited range of movement with only $10^{\circ}-90^{\circ}$ of knee flexion; further movements were restricted by pain. The arthroscopic portals were well healed. Plain radiographs showed no chondrocalcinosis. Although he was apyrexial, the inflammatory markers were raised, with the erythrocyte sedimentation rate and C-reactive protein levels at $110 \mathrm{~mm} / \mathrm{hr}$ (normal range, 
1 to $7 \mathrm{~mm} / \mathrm{hr}$ ) and $130 \mathrm{mg} / \mathrm{L}$ (normal range, 0.1 to $6 \mathrm{mg} / \mathrm{L}$ ), respectively. He underwent an arthroscopic joint washout of the knee. The graft was found to be intact (Fig. 2), and there was no chondrocalcinosis of the menisci. He had no new arthroscopic findings in terms of degenerative changes. The synovial fluid was straw-colored and grew no organisms or culture but was positive for calcium pyrophosphate crystals on microscopic examination. The fluid was obtained at the time of washout, as given the amount of swelling and raised inflammatory markers, it was considered more appropriate to improve the patient's symptoms even if the diagnosis proved not to be septic arthritis.

He was treated with non-steroidal anti-inflammatory drugs (Diclofenac) and his pain improved. Unfortunately, he did not follow the full postoperative physiotherapy rehabilitation and did not attend clinic appointments. One year later, he presented with

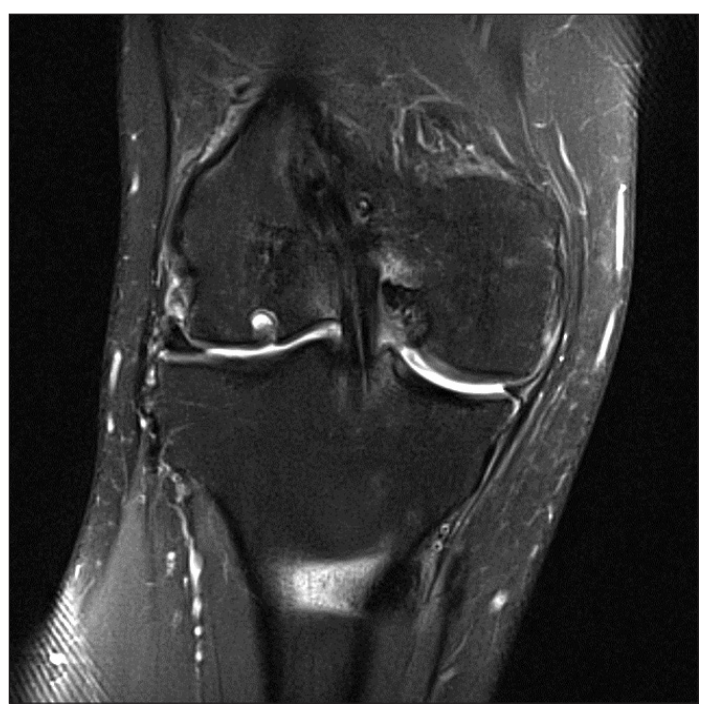

Fig. 1. Coronal magnetic resonance imaging scan of the knee showing abnormal bone in the lateral femoral condyle and the adjacent tibial plateau. continuing pain in the knee. A further arthroscopy at that time was performed to debride the knee and alleviate the pain, which showed the graft had incorporated well but degenerative changes had progressed to involve both the lateral and patellofemoral compartments (Fig. 3).

\section{Discussion}

Acute onset of pain following an ACL reconstruction can raise the possibility of infective arthritis. In our case, the knee was washed out and the synovial fluid was positive for pseudogout crystals.

Pseudogout is characterised by the deposition of CPPD crystals in the synovial fluid or menisci. It usually presents in one or multiple large joints, with severe joint pain, redness and swelling due

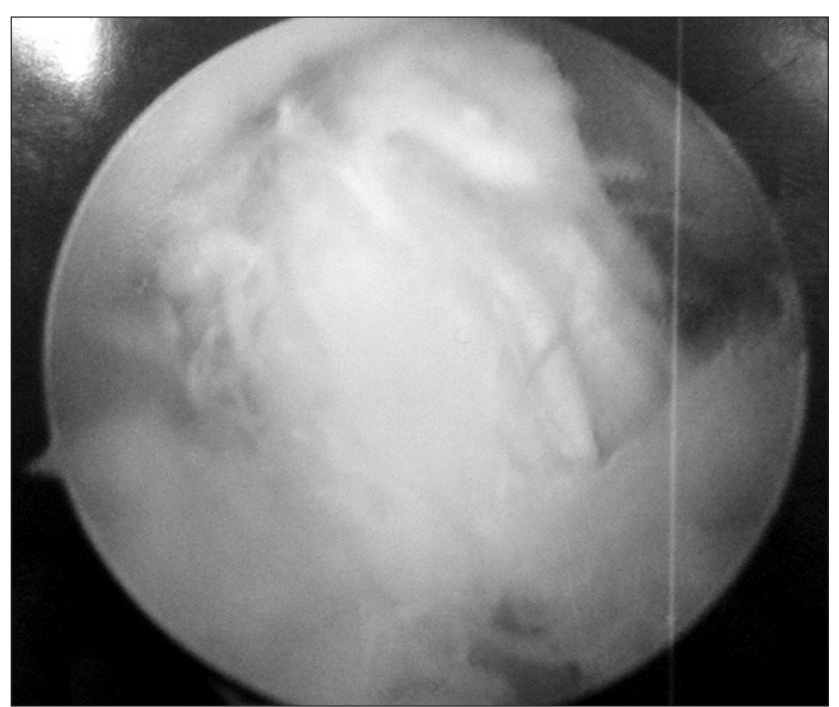

Fig. 2. Arthroscopic image obtained during knee washout after anterior cruciate ligament reconstruction showing the intact graft.
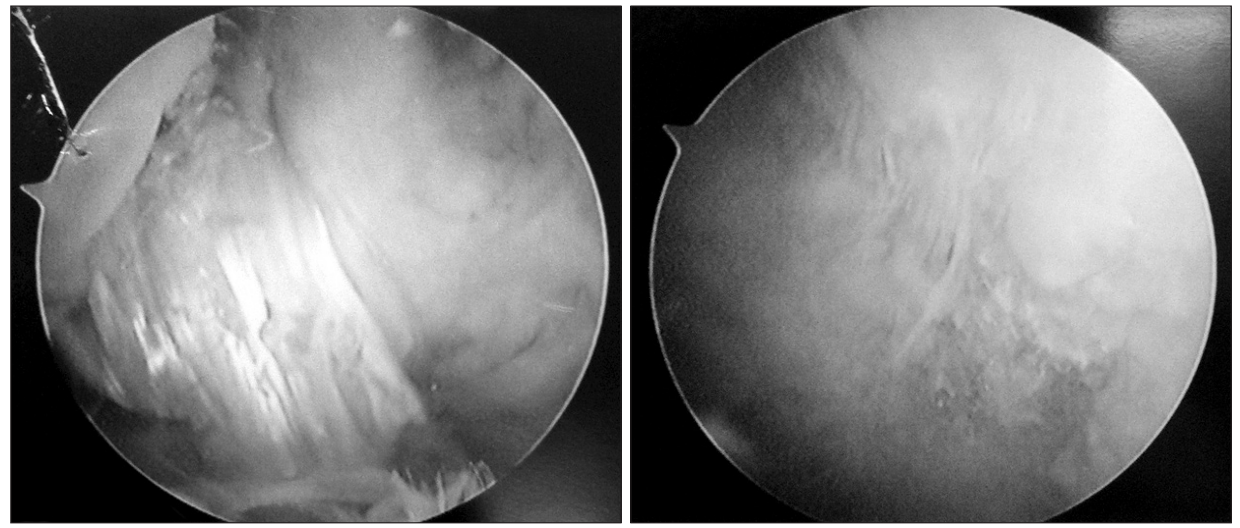

Fig. 3. Arthroscopic images obtained one year after anterior cruciate ligament (ACL) reconstruction showing the ACL graft fully incorporated and intact, and extensive cartilage loss over the trochlear region. 
Table 1. Comparison of Our Case with a Previously Reported Case of Pseudogout Following Anterior Cruciate Ligament Reconstruction

\begin{tabular}{lll}
\hline \multicolumn{1}{c}{ Variable } & \multicolumn{1}{c}{ Minezaki et al. ${ }^{2)}$} & \multicolumn{1}{c}{ Our case } \\
\hline Age $(\mathrm{yr})$ & 34 & Male \\
Sex & Female & Lateral meniscal tear, chondral changes in lateral \\
Knee observations & Medial meniscal tear & Hamstring tendons \\
Graft used & Leeds-Keio artificial ligament & Six weeks post-operative \\
Time of presentation & Seventeen months post-operative & Sudden onset of pain and swelling in the knee \\
Presentation & Pain and occasional effusion in the right knee & Microscopic joint fluid examination \\
Diagnosis method & Microscopic joint fluid examination, radiographs & Non-steroidal anti-inflammatory medications \\
Treatment & & -
\end{tabular}

to effusion ${ }^{1-4)}$.

Several causes of CPPD deposition have been proposed, with its occurrence being classified as either primary (non-traumatic) or secondary (traumatic). Primary deposits are thought to be due to a metabolic cause, such as hyperparathyroidism and haemochromatosis where crystallization occurs within the synovial fluid ${ }^{2,5,6)}$. Secondary deposits are more likely to occur in the presence of degenerative knee changes as a result of trauma and surgery ${ }^{2}$. It has been proposed that the mechanism for this is either that the crystals are liberated or 'shed' from preformed deposits within the joint or alternatively that an intra-articular insult may trigger their formation ${ }^{2,5)}$.

Symptoms of acute pseudogout are similar to that of septic arthritis ${ }^{1)}$, which poses diagnostic difficulties. Investigations such as joint aspiration, synovial fluid microscopic examination and microbiological culture as well as radiological imaging may help in the diagnosis ${ }^{1}$. Where doubt exists, arthroscopic washout of the knee is performed.

Pseudogout attack following an ACL reconstruction is a very rare event with only one previous report having been described (Table 1). Minezaki et al. ${ }^{2}$ described a 34-year-old female who was found to have calcification of the medial and lateral meniscus due to CPPD at 17 months after ACL reconstruction. Our report seems to be the first presentation of an acute pseudogout attack in the early postoperative period following ACL reconstruction. One can only postulate why such an attack occurs. We suggest that it may be that the trauma of surgery (including notch debridement and drilling of the femoral and tibial tunnels), when superimposed on pre-existing degenerative changes, can precipitate an acute attack, and that pseudogout attack may signify the progression of degenerative changes and a poorer outcome in the long-term.

In summary, pseudogout must be considered as a differential diagnosis of acute onset pain following ACL reconstruction, especially in a knee with pre-existing degenerative changes.

\section{Conflict of Interest}

No potential conflict of interest relevant to this article was reported.

\section{References}

1. Hirose CB, Wright RW. Calcium pyrophosphate dihydrate deposition disease (pseudogout) after total knee arthroplasty. J Arthroplasty. 2007;22:273-6.

2. Minezaki T, Tomatsu T, Hanada K. Calcium pyrophosphate dihydrate crystal deposition disease after anterior cruciate ligament reconstruction. Arthroscopy. 1998;14:634-6.

3. Ellman MH, Krieger MI, Brown N. Pseudogout mimicking synovial chondromatosis. J Bone Joint Surg Am. 1975; 57:863-5.

4. Longmore M, Wilkinson I. Rheumatology: crystal arthropathies. In: Longmore M, Wilkinson I, Turmezei T, Cheung CK, eds. Oxford handbook of clinical medicine. 7 th ed. Oxford: Oxford University Press; 2007. p534.

5. Doherty M, Dieppe PA. Acute pseudogout: "crystal shedding” or acute crystallization? Arthritis Rheum. 1981;24:954-7.

6. Resnik CS, Resnick D. Calcium pyrophosphate dihydrate crystal deposition disease. Curr Probl Diagn Radiol. 1982; 11:1-40. 